\title{
Reply to the Letter to the Editor of T.P. Schlösser et al. concerning "Pelvic incidence: an anatomic investigation of 880 cadaveric specimens" by Weinberg DS, Morris WZ, Gebhart JJ, Liu RW: Eur Spine J (2015); DOI 10.1007/s00586-015-4317-z
}

\author{
D. S. Weinberg ${ }^{1}$
}

Received: 1 December 2015/Revised: 1 December 2015/ Accepted: 1 December 2015/Published online: 10 December 2015

(C) Springer-Verlag Berlin Heidelberg 2015

We thank you for your interest and critical review of our manuscript [1]. We share your concerns regarding the reliabilities and potential limitations of conventional radiographic assessment of pelvic incidence, as were discussed in our manuscript as well as yours [2].

We too would agree that $3 \mathrm{D}$ analysis may provide more useful analyses in many settings, and share your enthusiasm for their potential for clinical use. As you mention, ex vivo $\mathrm{CT}$ shows equivalent reliability to osteological methods. Going forward, it is our hope that research such as yours will spark an increased clinical awareness and utility of pelvic incidence. Obviously, the osteological method we performed could not be performed in vivo, and CT may provide a more tangible intermediary. We suspect that this may be especially useful for practitioners evaluating concomitant pathologies of the hip, where CT imaging may have already been performed; we look forward to soon sharing our ongoing research in this area [3].

Regarding some of the methodological inquiries that were raised, we would like to remind readers that normality was appropriately accounted using the statistical methods described. Moreover, nonparametric analyses are rigorously more "liberal" and therefore more inclined to cause a type-I error. Therefore, readers should feel especially confident when interpreting a negative result in the present study that suggests no significant differences existed between the groups in question.

D. S. Weinberg

dsw56@case.edu

1 Division of Pediatric Orthopaedic Surgery, Case Western Reserve University, Rainbow Babies and Children's Hospital, 11100 Euclid Avenue, RBC 6081, Cleveland, $\mathrm{OH} 44106$, USA
In terms of selection bias, our cadaveric collection has no bias toward musculoskeletal disease nor abdominal or pelvic disease, but rather was created from unclaimed deceased bodies collected in a random fashion.

Finally, we would like to thank Schlosser et al. [2] for reporting their interesting results on pelvic incidence during growth. Since our study only included adult subjects, we were not able to comment on such, although studies such as yours as that of Mangione et al. [4] provide convincing evidence that pelvic incidence increases throughout growth until adolescence.

Compliance with ethical standards

Conflict of interest The author declares no conflicts of interest.

\section{References}

1. Weinberg DS, Morris WZ, Gebhart JJ, Liu RW (2015) Pelvic incidence: an anatomic investigation of 880 cadaveric specimens. Eur Spine J. doi:10.1007/s00586-015-4317-z

2. Schlosser TP, Janssen MM, Vrtovec T, Pernus F, Oner FC, Viergever MA, Vincken KL, Castelein RM (2014) Evolution of the ischio-iliac lordosis during natural growth and its relation with the pelvic incidence. Eur Spine J 23:1433-1441. doi:10.1007/ s00586-014-3358-z

3. Gebhart JJ, Bohl MS, Weinberg DS, Cooperman DR, Liu RW (2014) Pelvic incidence and acetabular version in slipped capital femoral epiphysis. J Pediatr Orthop. doi:10.1097/bpo. 0000000000000342

4. Mangione P, Gomez D, Senegas J (1997) Study of the course of the incidence angle during growth. Eur Spine J 6:163-167 